\title{
Emotion-Augmented Machine Learning: Overview of an Emerging Domain
}

\author{
Harald Strömfelt, Yue Zhang, Björn W. Schuller \\ GLAM, Department of Computing, Imperial College London, London, United Kingdom \\ Email: $\{$ h.stromfelt17; yue.zhang1; bjoern.schuller\}@imperial.ac.uk
}

\begin{abstract}
Emotion has been exposed as a crucial component of intelligent behaviour. Considerations in both neuroscience and psychology have identified emotion as playing a central role in various critical cognitive processes, such as attaining salience from the environment in order to support decision making, exploration-exploitation and broader adaptation. This paper provides an overview of some of the corroborative material in these fields, to then consider how emotion has been translated into machine learning. We identify emotion as being a promising endeavour for machine learning and expose Emotion-augmented Machine Learning (EML) as a frontier field in Artificial Intelligence and Affective Computing.
\end{abstract}

\section{Introduction}

The fascination of Artificial Intelligence (AI) is more than a practical one; we express a deeper affiliation with emotional AI, proclaimed by its portrayal in popular culture. In "ExMachina" (2014), a clear capability that differentiates this AI is her ability to use emotion as a means for manipulation in a Turing test. Whether she 'feels' real emotion as a manifestation of consciousness or simply analyses and simulates emotion is a philosophical issue. However, a different question could be asked - what is emotion to us humans? Is it simply a nuisance feature which clouds and inhibits good, rational, decision making, so that it becomes necessary for computers to understand our potentially illogical quirks? Or is it a necessity for our survival, playing a deeper role in how we learn to navigate an uncertain world?

Within the realm of Affective Computing (AC) [1] and its related fields (Social Signal Processing [2], multimodal affective behaviour generation for social robotics and virtual agents [3]-[5]), recognition and expression of emotions alone would enable unemotional, but affective computers to provide better performance in interacting with humans and decisionmaking [1], [6]. In the foundation of AC, Picard stops at a machine driven by its emotions. However, "driven by emotion" does not necessarily imply having a consciousness as being featured by strong AI, but it can also be referred to motivation, ideation, and curiosity [7].

Scientists have maintained that emotion plays an essential role in human perception, cognition, and action, as well as bodily processes [7], [8]. In particular, emotion constitutes a primary motivation engine [7] and is directly linked to the mammalian memory and learning system [9], [10], thus influencing reasoning, association, abstraction, and intuition. However, conventional machine learning algorithms are targeted at minimising some form of error function, where emotion as an incentive is completely absent. Conceivably, important parts of human emotional intelligence have to date remained understudied in $\mathrm{AC}$, but can be exploited for various goals of machine learning including learning efficiency (e.g., better convergence), computational cost, model confidence, and accuracy.

In this paper, we provide an overview of recent trends in the emerging field of Emotion-augmented Machine Learning (EML). To this end, we provide a précis of the emotion theory from a psychological and neuroscientific perspective and examine how these have been translated into ML algorithms.

\section{Mammalian Emotion}

\subsection{Psychological Basis of Emotion}

Emotion representations serve as psychological constructs for modelling, analysing, and recognising emotion, of which the most prevalent ones are derived from categorical, dimensional, and appraisal-based approaches [4]. The categorical emotion theory construes the emotion system as a limited set of discrete entities, such as the basic emotions [11]. On the other hand, advocates of the dimensional concept have modelled emotion as a multidimensional space continuum [12], [13], which is spanned by the valence (pleasant-unpleasant) and arousal (activation-deactivation) axes according to the Circumplex model [14]. Within the context of Reinforcement Learning [15], the appraisal paradigm, pioneered by Frijda [16] and Lazarus [17], forms the fundamental basis for various computational models [18].

Focusing on the aspect of emotion elicitation, contemporary appraisal theories are constituted on the premise that emotions are adaptive responses, which reflect evaluations of significant features of the environment for well-being [19][21]. Accordingly, emotion has been viewed as a rewardpunishment mechanism in brain function [22]. In each of these considerations, emotion acts as a guidance system, focusing attention to specifics within a complex environment and weighting their importance accordingly. Effectively, emotion can be categorised into two classes, primary and secondary [23]. Primary emotions are intrinsic reactions to environmental stimuli that are concerned with the needs of a system, e.g., the smell of food which is related to the 


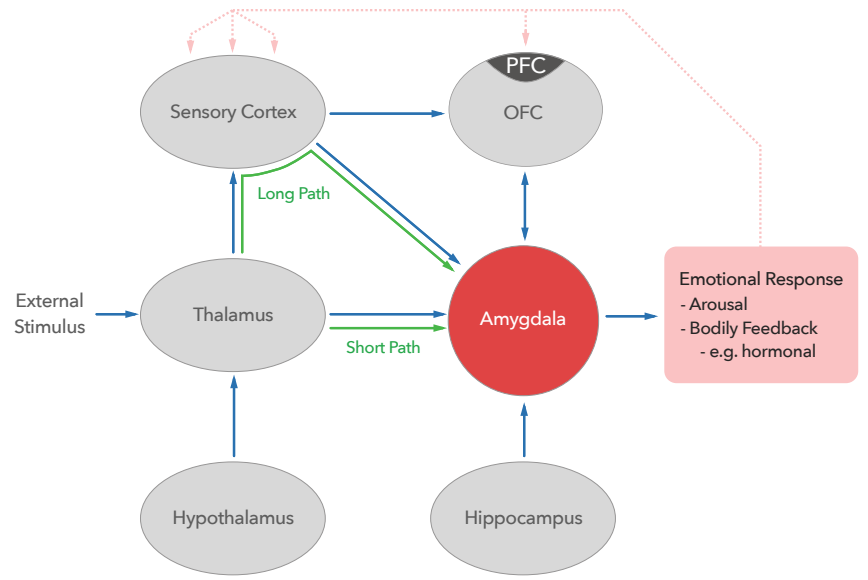

Figure 1. Schematic of amygdala interaction with other brain-systems. This is not an exact representation but it is one that, in various forms, has computational analogies (reviewed in Section 3) and aids in visualising the interconnectedness of the amygdala. The orbitofrontal cortex (OFC) is one constituent of the greater prefrontal cortex (PFC), but it should be noted that there are also other components. The dashed line represents the feedback effects of resultant emotional response. Adapted from [24]-[27].

need to eat. Secondary emotions arise from association with primary stimuli and are therefore associative emotions that are learned through, e.g., reinforcement. These associations then become learned predictors for when primary emotional response will occur [23].

\subsection{Emotion in the Brain}

To take inspiration from mammalian emotion, it is first necessary to gain a functional understanding on how emotion arises within the brain. Through neuroimaging studies, it has been possible to uncover some of the brain structures involved with emotion and shed light on how emotion fits into cognition. That said, the roles of each structure in the brain are not so easily separated. For this reason, emotion in the brain is multifaceted, wherein emotional details result from complex and intertwined activities between various brainsystems. This review is by no means exhaustive nor deeply focused on exact brain segments. The aim is to provide a mechanistic understanding for the separate brain-functions and how they intermingle to produce system-level behaviour.

It has long been thought that emotion is derived from the limbic system, a vast arrangement with commonly included areas such as the orbitofrontal cortex (OFC), which, together with other parts of the prefrontal cortex (PFC), is implicated in reinforcement and working memory; the hypothalamus, related to involuntary activity; the thalamus, junction for sensory information; the hippocampus, context and declarative memory; the sensory cortex, handling sensory information; parts of the basal ganglia, action control; and amygdala, processing positive and negative affect [9], [10], [23], [27]-[32]. However, the limbic system as a whole has gradually become dereferenced from emotion [30] and the responsibility of emotional function has shifted most consistently to the amygdala [29]. For instance, it has been shown that the amygdala is active in both positive and negative reward processing from stimuli [30], [31], [33][35].

Structurally speaking, the amygdala is highly connected within the brain, sharing inward and outward projection with the aforementioned limbic system components [31] as well as others through both direct and indirect affect [27]. Thus, the amygdala (and therefore emotion) is involved in multiple neuronal processes, such as the processing of raw (thalamus), object level (cortex), and contextual (hippocampus) data [29]. Further, by outward afference with the basal ganglia, the amygdala can also influence motor behaviour; and also is implicated with automatic responses (e.g., endocrine) via other systems [29], [33]. It should be noted that the amygdala itself is a complex formation consisting of many conjoined parts, with each giving rise to unclear but varied behaviour [23], [31], [34], [36]. However, in this the paper the amygdala shall be considered as a unitary structure.

The amygdala is thought to contribute to attentional behaviour, able to obtain salience from the environment. Models have approached the amygdala as an evaluator of stimuli which can shift focus towards emotionally associated features by way of indirect interaction with sensory systems, through arousal networks [30] such as the locus coeruleus (LC) [37], [38]; or through adjusting cognitive goals via projection with prefrontal cortices [39], [40].

The formation of emotional associations is known as emotional learning. Whereas the hippocampus and areas of the cortex are involved with declarative memory, which relates to memories that can be consciously remembered (sometimes referred to as explicit memory), the amygdala is likely responsible for storing stimuli-emotional response patterns [9], [27], [29], [30]. That said, the amygdala has also been shown to be participant in the formation of declarative memory. Firstly, it is understood to provide "emotional colouration" to declarative memories [29]. Secondly, emotional arousal provokes the secretion of the neuromodulator norepineprine (NE), via activation of the LC (sometimes referred to as the LC-NE system [37]), which induces plasticity in neurons and enhances memory and learning [34], [41]-[43]. Thus, emotional arousal coincides with enhanced vividness of declarative memory [27], [43], [44], which is confirmed by lesion and inhibitory studies of the amygdala that result in the obstruction of this process [30]. The evidence of the amygdala's (and by extension emotion's) role in attention and memory depicts emotion as being crucial in acquiring salience from the environment, picking out the most important stimuli with regards to survival [34], [35], [39], [45]-[47].

Studies analysing how the brain is able to traverse through exploration-exploitation modes have exposed two separate neuronal mechanisms that give rise to exploitation and exploration [48]-[54]. There are three important aspects to consider in this dilemma, the first is attention during exploration wherein the brain is seen to perform switching between options via attentional control, mediated perhaps by the LC-NE systems and prefrontal cortices [37], [38], [48]. The amygdala's influence on attention via the LC and prefrontal cortices therefore associates it with this 
system. On the other hand, reinforcement (both reward and punishment) processing is necessary during exploitation [48]. Reinforcement processing seems to occur in a number of regions: in the amygdala, establishing positive and negative associations from/with stimuli [29], [31], [34], [35], storing the variations in magnitude of reinforcement [36], [42], [55], encoding reinforcement associations [34], [56], processing reinforcement to learn reward-predicting cues and subsequently controlling arousal and response [31], [57]; in the OFC (and more generally the PFC), performing stimulus-reinforcement learning, correcting associations and controlling behaviours related to the reinforcement [28], [58] in relation to internal goals [54], also in preserving reinforcement information in working memory such as that which the amygdala generates [55], [59], representing the magnitude of reward [60] and in related cognitive action planning/selection [50], [51]; in the basal ganglia, where the likelihood of reinforcement is encoded [55]; in the striatal systems, on related habitual action-selection [50], [51] based on dopaminergic projections in response to expected reward-prediction [42], [53]. The amygdala-PFC interaction is notably interesting, since the amygdala responds to changes in outcome value and the PFC in action-planning/selection [36]; and potentially negative prediction error as well [61]. It is possible that this interaction is what allows generalisation and abstraction in humans [24]. Moreover, the amygdala might capture "state" value, where state refers to both internal concerns (e.g., needs such as thirst) and external stimuli or contexts; and that the understanding of valence via the amygdala is synonymous with that of value [62].

The importance of neuromodulators, such as NE, in reinforcement and, thereby, learning has been touched upon lightly. However, there are other neuromodulators that should also be given consideration, as the duties of neuromodulators are manifold and manifest themselves in many ways. By drawing from theoretical models and experimental data, Doya [63] sought to assimilate the different roles of the various neuromodulation systems on reinforcement learning, and describe them through associations with parameters used in computational Reinforcement Learning (RL) [63], [64]. In his model, dopamine signals reward prediction error, serotonin acts as a reward expectancy modulator, NE affects action-selection randomness and acetylcholine is associated with memory update speed. A discussion of each of these neuromodulators is not possible here, due to spatial restrictions, but in Doya's paper only acetylcholine is identified as a "major" projector to the amygdala. Contrarily, separate work has shown the amygdala to be notably afferent with dopamine, where, when tasked with cognitive exercises, the amount of dopamine in the human amygdala increased. Moreover, the levels observed correlated with learning performance [56]. As discussed, the amygdala has been connected with learning reward-prediction cues and identified as being sensitive to reinforcement variations, so it would seem that the dopamine model of Doya fits in with the amygdala profile depicted here. In any case, there is ample evidence that affirms the amygdala (and with it, emotion) as a centrepiece in neuroeconomic decision [62].

\section{Emotion-augmented Machine Learning}

Can the basis of the amygdala and emotion be forwarded into AI with positive consequence? Many of the leaps forward in AI have been drawn from associations with biology: Artificial Neural Networks (ANNs) themselves have been inspired by the biological neural network; and the Convolutional Neural Network (CNN) modelled after the visual cortex [65]. What about emotion? Emotion has, conceptually at least, begun to manifest itself in the realm of machines. As mentioned, AC has opened the door to analysis and synthesis, but the work of e.g., Morén [23] has lead to promising computer models which incorporate emotion concepts into the learning process. In this section, the different ways in which artificial emotion has been used in AI will be discussed, covering novel examples where emotion is intrinsic to the machine.

\subsection{Emotion for Optimisation Tasks}

In addition to analysing and synthesising emotions, emotion has been applied at a deeper level, where emotional concepts are adapted at the infrastructure level. Khashman [66] augmented a multilayer perception (MLP) with a somewhat psychological conceptualisation of "confidence" and "anxiety". The emotional backpropagation (EmBP) learning algorithm consists of an extra emotional bias that is applied to hidden and output layers. In a facial recognition task, its input is the global input pattern average value, $Y_{P A T}$, which aims to capture the global detail of each image (see [66] for its mathematical definition). During backpropagation, the emotional bias weight $W_{e m}^{i j}$, for hidden layer $j$ projecting to forward layer $i$, is updated with

$$
\Delta W_{e m}^{i j}=\mu \cdot \Delta_{e r r} \cdot Y_{P A T}+k \cdot\left[\delta W^{i j}(\text { old })\right],
$$

where $\mu$ and $k$ are the anxiety coefficient and confidence coefficient, respectively and vary between 1 and $0 ; \Delta_{e r r}$ is the backpropagated error from $i$ to $j$ and $\delta W^{i j}$ is the previous weight change for weights between layer $i$ and $j$. The anxiety coefficient is increased when new patterns are presented and in relation to output vs expected error in recognition (see [66]); the confidence coefficient is simply $1-\mu$. The effect of anxiety is therefore to enhance the inclusion of the latest error, as opposed to confidence which places dominance onto the previous updates. On two facial recognition experiments Khashman found that, compared with an MLP, the inclusion of these emotional parameters improved both recognition rate and execution time after training [66], [67]. The inclusion of anxiety, whereby it effectively slows learning on new tasks, can be seen as a sort of attentional feature, focusing learning on new and 'interesting' data.

In later work by Khashman on EmBP [68], impression was included (iEmBP). Conceptualised as a visual cue of a target object, which could either enhance or suppress anxiety depending on whether the impression was good or bad. The impressions were deduced through average image grey levels, such that a darker image is associated with a bad impression. iEmBP produced a higher recognition rate than both EmBP 
and MLP, in a blood cell type identification task, however, some other changes were also included such as attempting to capture 'memory' of patterns by using accumulated input patterns during each epoch [68].

In a separate model named DuoNN, Khashman introduced a new type of neuron. These "DuoNeurons" consist of a cognitive neuron, which processes local features, and an emotional neuron, which only considers global patterns [69]. This model was inspired by the visual cortex, splitting the data pathways into dorsal/cognitive and ventral/affective streams. Compared to EmBP, DuoNN produced higher recognition rates.

Furthering work on an optimisation algorithm inspired by chaos theory [70], which was found to converge onto local minima in an optimisation task, Yang et al. [71] showed that the injection of artificial emotion can aid in avoiding optima traps. This model is interesting as it portrays the role of emotion in exploration-exploitation and its artificial emotion generator included sensations, feelings, emotions and also a hormone system which would feed back into the activations of feelings [72]. Here, emotions are more abstract, where Khashman called them emotions they are here noted as 'sensations'. Included are: anxiety, triggered by diminishing reward; confidence, which, as with Khashman [66], responds oppositely to anxiety; fear, increases with anxiety and is implicated with strategy control; and warmth, whose function is to signal when to end the algorithm depending on fear and the iteration count. By modelling these sensations, a dominant emotion is generated, which is then applied as an executive affect, whereby it influences the algorithm parameters and can instigate strategy/behaviour change. Yang et al. [71] found that the use of artificial emotion increased convergence speed and outperformed competing methods.

Another model, EMotional Artificial Neural Networks (EMANN), augmented an MLP by adding neuromodulation by way of 'hormonal' glands [73]. These glands are managed by the neural network and are in fact nodes within the network. Therefore, nodes are both receptors and emitters of hormones. The hormones impact the neural network by editing the activation threshold, the summation process for adding up weights and also the output of a given node. In this model, weights are calculated with

$$
W_{i, j}(H)=\theta_{i, j}+\sum_{h} \phi_{i, j, h} H_{h},
$$

where $W_{i, j}$ is the weight on node $i$, from the $j$ th node in the previous layer, which constitutes the normal weight, $\theta_{i, j}$, in addition to a hormonal element. This element is the summation of responsiveness, $\phi_{i, j, h}$, on hormone level, $H_{h}$, where $H$ is the set of hormone levels and $h$ is a specific hormone. Further, to allow for hormonal influence on output, a proposed 'Hill-function' was used to calculate output. In testing, both the MLP and EMANN were evolved using a genetic algorithm and overall the EMANN was shown to be significantly more effective, whereby both would initially increase in performance but as the MLP would flat-line, the EMANN would continue to increase performance. This work suggests that the hormonal effects of emotion are an important consideration for AI [73].

\subsection{Anatomical Models in AI}

Whilst some models approach specific aspects of emotions, identifiable with how a person's feelings might change how they act, there has been work on holistic models which replicate brain systems [74], such as the limbic system. Morén \& Balkenius devised computational models, with modules representing the hippocampus, OFC, amygdala, thalamus and sensory cortex, as a means to produce emotional conditioning via the interdependencies of the components [23], [23], [75], [76]. The amygdala learns emotional associations, whilst the OFC acts as contextual inhibitor. Context is provided as an injection to the OFC from the hippocampus module, where it is generated by matching stimuli to locations. These pairings create an expectation for stimuli, which if unmet causes a shift in context [75].

The work of Morén and Balkenius laid down a framework for computation systems which leverage the amygdala and related brain systems; and has since been pioneered. Lucas et al. [77] created a limbic system inspired algorithm, BELBIC, which was used as a controller in control engineering tasks. Compared to a proportional-integral-derivative (PID) controller and optimised non-linear controllers, BELBIC was found to exhibit generalisation and flexibility, wherein the algorithm could appropriately adapt to parameter changes and disturbances. Incorporating the idea of short and long paths of stimuli to the amygdala [23], [25], [26], where the short path bridges the sensory thalamus-amygdala but the long path communicates via the frontal cortex (see Fig. 1), Babaie et al. [25] developed Brain Emotional Learning, BEL. By comparing amygdala output with input rewards the OFC either aligns the amygdala output to the reward if a reward is present, or inhibits the amygdala output if there is no reward. BEL outperformed both MLP and ANFIS - Adaptive Network based Fuzzy Inference System [78].

Lotfi \& Akbarzadeh-T [79] recently adapted the insights of Morén \& Balkenius in order to create their own, modelfree, AI algorithm. The Brain Emotional Learning-based Pattern Recogniser, BELPR, was found to outperform an MLP in both multi-input multi-output classification and chaotic time series prediction (see also [80] for a later version of BELPR, designed for online visual data processing). In another model, Lotfi \& Akbarzadeh-T introduced a forgetting process for the amygdala [26]. The model, Adaptive Decayed Brain Emotional Learning (ADBEL), uses a decay rate which, if fast, causes the amygdala weights to contain a lesser amount of their previous value on each update. This work was motivated by the need for a system to adapt to a changing environment and it was found that the model produced higher accuracy than its competitors, including ANFIS and MLP, and showed adaptability in an online prediction task. In another permutation, Lotfi \& Akbarzadeh-T added fuzzy logic to their ADBEL model [81], wherein the inputs and outputs of the amygdala and OFC were converted to fuzzy variables and evaluation was done with fuzzy operators. In this work, it was demonstrated that 'fuzziness' can boost the 
performance of learning, as in this case the convergence was faster than that of MLP and ADBEL; and that an emotional model can perform as a fuzzy learning algorithm.

Lotfi \& Akbarzadeh-T [82] then proposed LiAENN, the Limbic-Based Artificial Emotion Neural Network, which as the name suggests was modelled after the limbic system. In this approach, anxiety and confidence as emotions were used, together with short and long paths (see Figure 1), forgetting processes of the amygdala and emotion suppression via the OFC-amygdala interaction. This algorithm is a consolidation of EmBP, wherein anxiety and confidence and their attentional effects were used in learning; together with the brain interaction systems inspired models of Morén $\&$ Balkenius [23], Lucas et al. [77], Babaie et al. [25]. and the previous models of Lotfi \& Akbarzadeh-T. LiEANN was able to outperform the amygdala-OFC model of Morén \& Balkenius, as well as BELBIC, EmBP, DuoNN and BELPR. This work exemplifies the notion that emotionally derived concepts in AI can produce positive results, such that, seemingly, the inclusion of more emotional concepts leads to more positive results.

\subsection{Emotion in Computational Reinforcement Learning}

Emotion has begun to arise in computational Reinforcement Learning (RL) as a means to achieve more sophisticated behaviour than traditional RL, examples of which are surveyed thoroughly in [18]. In this overview, we remark on some of the key themes and relevant work, outlined by [18].

There have been a variety of approaches to fusing emotion and computational Reinforcement Learning [72], [83]-[85], wherein an agent must learn an optimal stateaction mapping policy such that the greatest reward is achieved [15]. One such approach is derived from Appraisal Theory, where appraisals are performed on the state and rewards are accordingly modified. A key adaptation for these methods is the conception of both intrinsic motivation (associated with internal or emotionally derived reward) and extrinsic motivation (externally applied reward as is common in standard RL), in the reinforcement reward [86], [87]. One example, by Marinier \& Laird [88], showed that with the incorporation of appraisal-induced emotion, it is possible to speed up learning compared to a standard RL agent. This was attributed to the fact that, in this case, emotion provides constant reward whereas in the standard RL agent, reward is only received at the end of each run. Further, by adding in 'Mood', wherein emotion was sustained during each run, learning became faster yet. This was because some necessary actions would cause states to receive no emotional response. Without the 'Mood' effect, these states might become undervalued but by persisting the emotional reward, state values would be better approximated. Other appraisal theory-based emotional methods have also shown similarly superior results compared to non-emotional RL [89], [90].

Alternatively, some models of emotional RL include the notion of homeostasis and drive, that is, the attraction towards an equilibrium state of an agent. In these models, homeostasis describes an equilibrium state for the agent with respect to homeostatic (or salient) variables, e. g., hunger and thirst (cf. primary emotions). The agent will experience drives, which produce actions that alter the agent's state in homeostatic space, towards the equilibrium; these changes can then be translated into rewards [91]-[93], note that 'Hullian' drive has also been used, which is similar but adopts a different approach to drive-induced behaviour [94].

Furthermore, emotion and other neuro-inspired principles have been incorporated in action-selection [95]-[97]. For example, valence has been used to influence explorationexploitation, where negative or positive valence promotes more or less considered evaluation in action-selection, respectively [98]. In another example, frustration was used to dictate which strategy or drive should be considered when deciding on an action (e.g., switching between using odometry navigation and visual navigation or prioritising a thirst vs hunger drive), in a robotics foraging experiment [99]. In a similar fashion, employing frustration as part of an amygdala model in Q-learning (see [64]) was seen to improve the navigation performance of an agent [100], whereby emotion would accordingly alter the weights of different value functions for selecting actions. For a more extensive survey on these topics see [18].

\subsection{Cognition and Abstraction in Learning}

In a (simulated) robotics application Parisi \& Petrosino [101] found that the addition of an 'emotional circuit' to a neural network-controlled agent allowed it to embody motivational decision-making. The neural network architecture was created with a genetic algorithm and it was found that robots that included emotion would achieve higher fitness when tested in a number of environments and with differing motivators. For example, some would have to look for both food and water, and the environment would provide varying amounts of each. In one case, food provisions would be five times greater than water. This lead to the robots placing a higher motivational priority on water, only seeking food when close to it or very hungry. In what Velásquez called Emotion-Based Control, a novel approach to action selection was developed, wherein emotional associations in learning would guide later decisions via bias signals. By using the Cathexis model, which comprises a set of systems, including an 'Emotion Generator', 'Behaviour Systems' and 'Motor Systems' [102]-[104], Velásquez was able to demonstrate emotional conditioning within a robotic agent called 'Yuppy'. Velásquez coupled a learning capability alongside some primary rules for emotional elicitation, and found that Yuppy would build emotional associations overtime. In one example, where Yuppy would already associate being 'disciplined' as a fear elicitor and where its cower would prompt Yuppy to make a sound, a connection would form between the sound and fear, where the two initially had no cognitive association. In a different case, Yuppy would, by primary rule, become happy upon finding a pink bone. However, if a person was carrying the bone and disciplined the robot, Yuppy would become wary of humans in later tests. In another, termed, 
cognitive architecture, the learning intelligence distribution agent (LIDA) also sought to produce cognitive behaviour, by incorporating attention, action-selection and motivation with emotion. Emotions are generated as the result of appraisals on events (producing emotional association), which then motivate different actions. In LIDA, emotion also enhances learning through arousal, but if arousal gets too great it will instead cause interferences [105]. One cannot help but envision these ground-breaking approaches as an abstractive reinforcement model, wherein initial primitive rules can be abstracted such that new behaviours are generated; and the understanding of good vs bad behaviour is much more sophisticated. This would have obvious connotations with both Appraisal Theory and also the notion that emotion works as to facilitate the satisfaction of an organism's core needs, producing new goals as fits those needs.

\section{Closing Remarks - What Comes Next?}

The brain shows us that reinforcement is not a unitary concept, and is more complex than simply knowing the actual reward associated with an action and then choosing actions accordingly. Instead elements such as expected reinforcement, reinforcement type (reward or punishment) and reinforcement prediction error are all separate entities with separate brain-systems dedicated to them. Lotfi and others showed us that promoting emotion from its role in Affective Computing, wherein it serves the human-computer interaction, to a construct with deeper affinity to AI can produce promising results. Innovative applications of emotion and related theories in RL demonstrate the broad range of new emotion-inspired algorithms and their potential with respect to the current state-of-the-art. In a similar fashion, Velásquez has shown us that allowing for emotional association, which could be synonymous with learning to understand and predict reinforcement, coupled with some primary emotion inducers, can produce adaptive and unplanned behaviour; LIDA has in turn showed us that other, cognitive, salient behaviour can indeed be captured with the application of emotion. All of this illustrates the effectiveness of emotionally augmented machine learning, wherein emotion acts as the guidance system for machines to traverse through a complex world in order to perform proportionally complex tasks.

\section{Acknowledgments}

The research work has received funding from the EPSRCDTA Training Grant, project reference EP/L504786/1.

\section{References}

[1] R. W. Picard, Affective Computing. MIT press Cambridge, 1997, vol. 252.

[2] A. Vinciarelli, M. Pantic, and H. Bourlard, "Social Signal Processing: Survey of an emerging domain," Image and Vision Computing, vol. 27, no. 12, pp. 1743-1759, 2009.

[3] M. Schröder, "Emotional speech synthesis: A review," in Proc. of INTERSPEECH. Aalborg, Denmark: ISCA, 2001, pp. 561-564.

[4] H. Gunes, B. Schuller, M. Pantic, and R. Cowie, "Emotion representation, analysis and synthesis in continuous space: A survey," in Proc. of International Conference on Automatic Face \& Gesture Recognition. Santa Barara, CA: IEEE, 2011, pp. 827-834.
[5] M. Pantic, V. Evers, M. Deisenroth, L. Merino, and B. Schuller, "Social and affective robotics tutorial," in Proc. of ACM Multimedia. Amsterdam, The Netherlands: ACM, 2016, pp. 1477-1478.

[6] J. Bates, "The role of emotion in believable agents," Communications of the ACM, vol. 37, no. 7, pp. 122-125, 1994.

[7] C. E. Izard, Human Emotions. Springer Science \& Business Media, 2013.

[8] B. L. Fredrickson, "What good are positive emotions?" Review of General Psychology, vol. 2, no. 3, pp. 300-319, 1998.

[9] J. E. LeDoux, "Emotional memory systems in the brain," Behavioural Brain Research, vol. 58, no. 1, pp. 69-79, 1993.

[10] J. LeDoux, The Emotional Brain: The Mysterious Underpinnings of Emotional Life. Simon \& Schuster, 1998.

[11] P. Ekman, "An argument for basic emotions," Cognition \& Emotion, vol. 6, no. 3-4, pp. 169-200, 1992.

[12] J. A. Russell and L. F. Barrett, "Core affect, prototypical emotional episodes, and other things called emotion: Dissecting the elephant," Journal of Personality and Social Psychology, vol. 76, no. 5, pp. 805-819, 1999.

[13] J. Jia, S. Zhang, F. Meng, Y. Wang, and L. Cai, "Emotional audiovisual speech synthesis based on pad," Transactions on Audio, Speech, and Language Processing, vol. 19, no. 3, pp. 570-582, 2011.

[14] J. A. Russell, "A Circumplex Model of Affect," Personality and Social Psychology, vol. 39, pp. 1161-1178, 1980.

[15] R. S. Sutton and A. G. Barto, Reinforcement Learning: An introduction. The MIT Press, 1998, vol. 1, no. 1.

[16] N. H. Frijda, P. Kuipers, and E. Ter Schure, "Relations among emotion, appraisal, and emotional action readiness," Journal of Personality and Social Psychology, vol. 57, no. 2, pp. 212-228, 1989.

[17] R. S. Lazarus, "Progress on a cognitive-motivational-relational theory of emotion," American Psychologist, vol. 46, no. 8, pp. 819-834, 1991.

[18] T. M. Moerland, J. Broekens, and C. Jonker, "Emotion in reinforcement learning agents and robots: a survey," Machine Learning Journal, 2017, to appear.

[19] A. Moors, P. C. Ellsworth, K. R. Scherer, and N. H. Frijda, "Appraisal theories of emotion: State of the art and future development," Emotion Review, vol. 5, no. 2, pp. 119-124, 2013.

[20] K. R. Scherer, "Appraisal considered as a process of multilevel sequential checking," Appraisal Processes in Emotion: Theory, Methods, Research, vol. 92, no. 92-120, p. 57, 2001.

[21] R. Lowe, C. Herrera, A. Morse, and T. Ziemke, "The embodied dynamics of emotion, appraisal and attention," Attention in Cognitive Systems. Theories and Systems From an Interdisciplinary Viewpoint, pp. 1-20, 2007.

[22] E. T. Rolls, "Précis of the brain and emotion," Behavioural and Brain Sciences, vol. 23, pp. 177-191, 2000.

[23] C. Morén and J. Balkenius, "Emotional learning: A computational model of the amygdala," Cybernetics \& Systems, vol. 32, no. 6, pp. 611-636, 2001.

[24] M. A. Arbib and J.-M. Fellous, "Emotions: From brain to robot," Trends in Cognitive Sciences, vol. 8, no. 12, pp. 554-561, 2004.

[25] T. Babaie, R. Karimizandi, and C. Lucas, "Learning based brain emotional intelligence as a new aspect for development of an alarm system," Soft Computing - A Fusion of Foundations, Methodologies and Applications, vol. 12, no. 9, pp. 857-873, 2008.

[26] E. Lotfi and M.-R. Akbarzadeh, "Adaptive brain emotional decayed learning for online prediction of geomagnetic activity indices," Neurocomputing, vol. 126, pp. 188-196, 2014. 
[27] K. S. LaBar and R. Cabeza, "Cognitive neuroscience of emotional memory," Nature Reviews Neuroscience, vol. 7, no. 1, pp. 54-64, 2006.

[28] E. T. Rolls, "The functions of the orbitofrontal cortex," Brain and Cognition, vol. 55, no. 1, pp. 11-29, 2004.

[29] J.-M. Fellous, J. L. Armony, and J. E. Ledoux, "Emotional circuits and computational neuroscience," Neuroscience, vol. 454, no. 7200, pp. $1-8,2002$.

[30] J. E. LeDoux, "Emotion circuits in the brain," Annual Review of Neuroscience, vol. 23, no. 1, pp. 155-184, 2000.

[31] E. A. Murray, "The amygdala, reward and emotion," Trends in Cognitive Sciences, vol. 11, no. 11, pp. 489-497, 2007.

[32] A. M. Graybiel, "The basal ganglia: Learning new tricks and loving it," Current Opinion in Neurobiology, vol. 15, no. 6, pp. 638-644, 2005.

[33] T. Dalgleish, "The emotional brain," Nature Reviews Neuroscience, vol. 5, no. 7, pp. 583-589, 2004.

[34] P. Namburi, A. Beyeler, S. Yorozu, G. G. Calhoon, S. A. Halbert, R. Wichmann, S. S. Holden, K. L. Mertens, M. Anahtar, A. C. Felix-Ortiz et al., "A circuit mechanism for differentiating positive and negative associations," Nature, vol. 520, no. 7549, pp. 675-678, 2015 .

[35] A. Beyeler, P. Namburi, G. F. Glober, C. Simonnet, G. G. Calhoon, G. F. Conyers, R. Luck, C. P. Wildes, and K. M. Tye, "Divergent routing of positive and negative information from the amygdala during memory retrieval," Neuron, vol. 90, no. 2, pp. 348-361, 2016.

[36] P. H. Janak and K. M. Tye, "From circuits to behaviour in the amygdala," Nature, vol. 517, no. 7534, pp. 284-292, 2015.

[37] G. Aston-Jones and J. D. Cohen, "An integrative theory of locus coeruleus-norepinephrine function: Adaptive gain and optimal performance," Annual Review of Neuroscience, vol. 28, pp. 403-450, 2005.

[38] S. J. Sara, "The locus coeruleus and noradrenergic modulation of cognition," Nature Reviews Neuroscience, vol. 10, no. 3, pp. 211-223, 2009.

[39] J. G. Taylor and N. F. Fragopanagos, "The interaction of attention and emotion," Neural Networks, vol. 18, no. 4, pp. 353-369, 2005.

[40] M. Gallagher and P. C. Holland, "The amygdala complex: Multiple roles in associative learning and attention," in Proc. of National Academy of Sciences, vol. 91, no. 25. National Academy of Sciences, 1994, pp. 11771-11776.

[41] H. Hu, E. Real, K. Takamiya, M.-G. Kang, J. Ledoux, R. L. Huganir, and R. Malinow, "Emotion enhances learning via norepinephrine regulation of AMPA-receptor trafficking," Cell, vol. 131, no. 1, pp. 160-173, 2007.

[42] J. Li, D. Schiller, G. Schoenbaum, E. A. Phelps, and N. D. Daw, "Differential roles of human striatum and amygdala in associative learning," Nature Neuroscience, vol. 14, no. 10, pp. 1250-1252, 2011.

[43] K. Tully and V. Y. Bolshakov, "Emotional enhancement of memory: How norepinephrine enables synaptic plasticity," Molecular Brain, vol. 3 , no. 15 , p. 9 pages, 2010 .

[44] J. L. McGaugh, "The amygdala modulates the consolidation of memories of emotionally arousing experiences," Annual Review of Neuroscience, vol. 27, pp. 1-28, 2004.

[45] E. A. Kensinger, "Negative emotion enhances memory accuracy behavioral and neuroimaging evidence," Current Directions in Psychological Science, vol. 16, no. 4, pp. 213-218, 2007.

[46] E. A. Phelps and J. E. LeDoux, "Contributions of the amygdala to emotion processing: From animal models to human behavior," Neuron, vol. 48, no. 2, pp. 175-187, 2005.
[47] A. Bechara and A. R. Damasio, "The somatic marker hypothesis: A neural theory of economic decision," Games and Economic Behavior, vol. 52, no. 2, pp. 336-372, 2005.

[48] D. Laureiro-Martínez, S. Brusoni, N. Canessa, and M. Zollo, "Understanding the exploration-exploitation dilemma: An fMRI study of attention control and decision-making performance," Strategic Management Journal, vol. 36, no. 3, pp. 319-338, 2015.

[49] D. Laureiro-Martínez, N. Canessa, S. Brusoni, M. Zollo, T. Hare, F. Alemanno, and S. F. Cappa, "Frontopolar cortex and decisionmaking efficiency: Comparing brain activity of experts with different professional background during an exploration-exploitation task," Frontiers in Human Neuroscience, vol. 7, no. Article ID 927, 2014.

[50] N. D. Daw, Y. Niv, and P. Dayan, "Uncertainty-based competition between prefrontal and dorsolateral striatal systems for behavioral control," Nature Neuroscience, vol. 8, no. 12, pp. 1704-1711, 2005.

[51] N. D. Daw, J. P. O'doherty, P. Dayan, B. Seymour, and R. J. Dolan, "Cortical substrates for exploratory decisions in humans," Nature, vol. 441, no. 7095, pp. 876-879, 2006.

[52] S. M. McClure, G. S. Berns, and P. R. Montague, "Temporal prediction errors in a passive learning task activate human striatum," Neuron, vol. 38, no. 2, pp. 339-346, 2003.

[53] B. W. Balleine, M. R. Delgado, and O. Hikosaka, "The role of the Dorsal Striatum in reward and decision-making," Neuroscience, vol. 27 , no. 31, pp. 8161-8165, 2007.

[54] E. K. Miller and J. D. Cohen, "An integrative theory of prefrontal cortex function," Annual Review of Neuroscience, vol. 24, no. 1, pp. $167-202,2001$

[55] M. J. Frank and E. D. Claus, "Anatomy of a decision: Striatoorbitofrontal interactions in reinforcement learning, decision making, and reversal," Psychological Review, vol. 113, no. 2, pp. 300-326, 2006.

[56] I. Fried, C. L. Wilson, J. W. Morrow, K. A. Cameron, E. D. Behnke, L. C. Ackerson, and N. T. Maidment, "Increased dopamine release in the human amygdala during performance of cognitive tasks," Nature neuroscience, vol. 4, no. 2, pp. 201-206, 2001.

[57] R. N. Cardinal, J. A. Parkinson, J. Hall, and B. J. Everitt, "Emotion and motivation: The role of the Amygdala, Ventral Striatum, and Prefrontal Cortex," Neuroscience \& Biobehavioral Reviews, vol. 26, no. 3, pp. 321-352, 2002.

[58] J.-M. Fellous, "Emotion: Computational modeling," Encyclopedia of Neuroscience, vol. 3, pp. 909-913, 2010.

[59] B. Knutson, G. W. Fong, S. M. Bennett, C. M. Adams, and D. Hommer, "A region of mesial prefrontal cortex tracks monetarily rewarding outcomes: Characterization with rapid event-related fMRI," Neuroimage, vol. 18, no. 2, pp. 263-272, 2003.

[60] J. O’Doherty, M. L. Kringelbach, E. T. Rolls, J. Hornak, and C. Andrews, "Abstract reward and punishment representations in the human orbitofrontal cortex," Nature Neuroscience, vol. 4, no. 1, pp. 95-102, 2001.

[61] A. A. Moustafa, M. W. Gilbertson, S. P. Orr, M. M. Herzallah, R. J. Servatius, and C. E. Myers, "A model of amygdala-hippocampalprefrontal interaction in fear conditioning and extinction in animals," Brain and Cognition, vol. 81, no. 1, pp. 29-43, 2013.

[62] S. E. Morrison and C. D. Salzman, "Re-valuing the amygdala," Current Opinion in Neurobiology, vol. 20, no. 2, pp. 221-230, 2010.

[63] K. Doya, "Metalearning, neuromodulation, and emotion," in Proc. of the Toyota Conference Conference on Affective Minds. Shizuoka, Japan: Elsevier, 2000, pp. 46-47.

[64] R. S. Sutton and A. G. Barto, "Reinforcement Learning: An introduction," p. 334 pages, 2011.

[65] Y. LeCun, Y. Bengio, and G. Hinton, "Deep Learning," Nature, vol. 521, no. 7553, pp. 436-444, 2015. 
[66] A. Khashman, "A modified backpropagation learning algorithm with added emotional coefficients," Transactions on Neural Networks, vol. 19, no. 11, pp. 1896-1909, 2008.

[67] _ "Application of an emotional neural network to facial recognition," Neural Computing and Applications, vol. 18, no. 4, pp. 309-320, 2009.

[68] _ "An emotional system with application to blood cell type identification," Transactions of the Institute of Measurement and Control, vol. 34, no. 2-3, pp. 125-147, 2012.

[69] — "Modeling cognitive and emotional processes: A novel neural network architecture," Neural Networks, vol. 23, no. 10, pp. 11551163, 2010.

[70] B. L. W. Jiang, "Optimizing complex functions by chaos search," Cybernetics \& Systems, vol. 29, no. 4, pp. 409-419, 1998.

[71] Y. Yang, Y. Wang, X. Yuan, and F. Yin, "Hybrid chaos optimization algorithm with artificial emotion," Applied Mathematics and Computation, vol. 218, no. 11, pp. 6585-6611, 2012.

[72] S. C. Gadanho and J. Hallam, "Robot learning driven by emotions," Adaptive Behavior, vol. 9, no. 1, pp. 42-64, 2001.

[73] R. Thenius, P. Zahadat, and T. Schmickl, "EMANN - a model of emotions in an artificial neural network," in Proc. of European Conference on Artificial Life, vol. 12, Taurmina, Italy, 2013, pp. 830-837.

[74] N. Navarro-Guerrero, R. Lowe, and S. Wermter, "A neurocomputational amygdala model of auditory fear conditioning: A hybrid system approach," in Proc. of International Joint Conference on Neural Networks (IJCNN). Brisbane, Australia: IEEE, 2012, pp. $1-8$.

[75] C. Balkenius and J. Morén, "A computational model of context processing," in Proc. of International Conference on the Simulation of Adaptive Behaviour. Paris, France: The MIT Press, 2000, pp. 256-265.

[76] J. Morén and C. Balkenius, "A computational model of emotional learning in the amygdala," From Animals to Animats, vol. 6, pp. 115-124, 2000.

[77] C. Lucas, D. Shahmirzadi, and N. Sheikholeslami, "Introducing BELBIC: Brain emotional learning based intelligent controller," Intelligent Automation \& Soft Computing, vol. 10, no. 1, pp. 11-21, 2004.

[78] J.-S. R. Jang, "ANFIS: Adaptive-network-based fuzzy inference system," Transactions on Systems, Man, and Cybernetics, vol. 23, no. 3, pp. 665-685, 1993.

[79] E. Lotfi and M.-R. Akbarzadeh-T, "Brain emotional learning-based pattern recognizer," Cybernetics and Systems, vol. 44, no. 5, pp. 402-421, 2013.

[80] E. Lotfi, S. Setayeshi, and S. Taimory, "A neural basis computational model of emotional brain for online visual object recognition," Applied Artificial Intelligence, vol. 28, no. 8, pp. 814-834, 2014.

[81] E. Lotfi and M.-R. Akbarzadeh-T, "Emotional brain-inspired adaptive fuzzy decayed learning for online prediction problems," in Proc. of International Conference on Fuzzy Systems. Hyderabad, India: IEEE, 2013, pp. 1-7.

[82] - "Practical emotional neural networks," Neural Networks, vol. 59, pp. 61-72, 2014.

[83] J. Broekens, E. Jacobs, and C. M. Jonker, "A reinforcement learning model of joy, distress, hope and fear," Connection Science, vol. 27, no. 3, pp. 215-233, 2015.

[84] S. C. Gadanho and J. Hallam, "Emotion-triggered learning in autonomous robot control," Cybernetics \& Systems, vol. 32, no. 5, pp. 531-559, 2001.

[85] R. Von Haugwitz, Y. Kitamura, and K. Takashima, "Modulating reinforcement-learning parameters using agent emotions," in Proc. of Joint International Conference on Soft Computing and Intelligent Systems (SCIS) and International Symposium on Advanced Intelligent Systems (ISIS). IEEE, 2012, pp. 1281-1285.
[86] S. Singh, R. L. Lewis, A. G. Barto, and J. Sorg, "Intrinsically motivated reinforcement learning: An evolutionary perspective," Transactions on Autonomous Mental Development, vol. 2, no. 2, pp. 70-82, 2010.

[87] S. P. Singh, A. G. Barto, and N. Chentanez, "Intrinsically motivated reinforcement learning," in Proc. of NIPS, vol. 17, no. 2, Vancouver, Canada, 2004, pp. 1281-1288.

[88] R. P. Marinier and J. E. Laird, "Emotion-driven reinforcement learning," in Proc. of the Annual Meeting of the Cognitive Science Society, vol. 30, no. 30. Washington, DC: Cognitive Science Society, 2008.

[89] P. Sequeira, F. Melo, and A. Paiva, "Emotion-based intrinsic motivation for reinforcement learning agents," Affective Computing and Intelligent Interaction, pp. 326-336, 2011.

[90] P. Sequeira, F. S. Melo, and A. Paiva, "Learning by appraising: An emotion-based approach to intrinsic reward design," Adaptive Behavior, vol. 22, no. 5, pp. 330-349, 2014.

[91] I. Cos, L. Cañamero, G. M. Hayes, and A. Gillies, "Hedonic value: Enhancing adaptation for motivated agents," Adaptive Behavior, vol. 21, no. 6, pp. 465-483, 2013.

[92] E. Coutinho, E. R. Miranda, and A. Cangelosi, "Towards a model for embodied emotions," in Proc. of Portuguese Conference on Artificial Intelligence (EPIA). Covilha, Portugal: IEEE, 2005, pp. 54-63.

[93] D. Cañamero, "Modeling motivations and emotions as a basis for intelligent behavior," in Proc. of International Conference on Autonomous Agents. Marina del Rey, CA: ACM, 1997, pp. 148-155.

[94] G. Konidaris and A. Barto, "An adaptive robot motivational system," in Proc. of International Conference on Simulation of Adaptive Behavior, Aberystwyth, UK, 2006, pp. 346-356.

[95] J. Broekens, W. A. Kosters, and F. J. Verbeek, "On affect and selfadaptation: Potential benefits of valence-controlled action-selection," in Proc. of International Work-Conference on the Interplay Between Natural and Artificial Computation, La Manga del Mar Menor, Spain, 2007, pp. 357-366.

[96] O. Avila-Garcia and L. Cañamero, "Using hormonal feedback to modulate action selection in a competitive scenario," From Animals to Animats, vol. 8, pp. 243-252, 2004.

[97] R. Lowe and E. Billing, "Affective-associative two-process theory: A neural network investigation of adaptive behaviour in differential outcomes training," Adaptive Behavior, vol. 25, no. 1, pp. 5-23, 2017.

[98] J. Broekens, W. A. Kosters, and F. J. Verbeek, "Affect, anticipation, and adaptation: Affect-controlled selection of anticipatory simulation in artificial adaptive agents," Adaptive Behavior, vol. 15, no. 4, pp. 397-422, 2007.

[99] C. Hasson, P. Gaussier, and S. Boucenna, "Emotions as a dynamical system: the interplay between the meta-control and communication function of emotions," Paladyn, vol. 2, no. 3, pp. 111-125, 2011.

[100] D. D. Tsankova, "Emotionally influenced coordination of behaviors for autonomous mobile robots," in Proc. of International IEEE Symposium Intelligent Systems, vol. 1. Varna, Bulgaria: IEEE, 2002, pp. 92-97.

[101] D. Parisi and G. Petrosino, "Robots that have emotions," Adaptive Behavior, vol. 18, no. 6, pp. 453-469, 2010.

[102] J. D. Velásquez, "Modeling emotion-based decision-making," Emotional and Intelligent: The Tangled Knot of Cognition, pp. 164-169, 1998.

[103] - "When robots weep: Emotional memories and decision-making," in Proc. of AAAI/IAAI. Madison, WI: American Association for Artificial Intelligence, 1998, pp. 70-75.

[104] - "Modeling emotions and other motivations in synthetic agents," in Proc. of AAAI/IAAI. Providence, Rhode Island: AAAI, 1997, pp. $10-15$.

[105] S. Franklin, T. Madl, S. D’Mello, and J. Snaider, “LIDA: A systemslevel architecture for cognition, emotion, and learning," Transactions on Autonomous Mental Development, vol. 6, no. 1, pp. 19-41, 2014. 